\title{
Pre-vaccination immune response to COVID-19 in a population in Northeast Portugal
}

\author{
Mary Duro ${ }^{1,2,3} \cdot$ Inês Duro ${ }^{4} \cdot$ Irene Rebelo ${ }^{5,6} \cdot$ Filipa Moreno $^{2} \cdot$ Manuel Pires $^{2} \cdot$ Sofia Jacinto $^{2} \cdot$ Marilda Pimentel $^{2}$. \\ Cristina Maria Nunes Almeida ${ }^{1}$ (])
}

Received: 4 June 2021 / Accepted: 28 September 2021 / Published online: 12 October 2021

(c) The Author(s), under exclusive licence to Royal Academy of Medicine in Ireland 2021

\begin{abstract}
Purpose To study the immunization status and IgM and IgG antibody behavior against severe acute respiratory syndrome coronavirus 2 (SARS-CoV-2) in an unvaccinated population of Northeast Portugal (including RT-PCR diagnosed and undiagnosed individuals).

Methods Application of a clinical-epidemiological survey, and analysis of IgM and IgG SARS-COV-2 antibodies (against $\mathrm{N}$ core protein) in 362 participants that voluntarily sought the laboratory for testing.

Results At the time of the analysis, $31.7 \%(n=114)$ of the study population had a previous SARS-CoV-2 diagnosis, $48.3 \%$ of which were asymptomatic, and $71.9 \% \mathrm{IgG}$ seropositive. Of these, $83.3 \%$ and $60 \%$ were, respectively, $\operatorname{IgM}$ and $\operatorname{IgG}$ seropositive within 2 weeks after the initial diagnosis. Both antibodies peaked in the 3 rd week post diagnosis, with titers decreasing over the following weeks, until a state of seronegativity was achieved after the 6th week for IgM, and the 21 st for IgG. Symptomatic patients showed higher IgM and IgG values, when compared to asymptomatic ones. Fever, the most reported symptom, was found to be positively associated with IgM values. Ages of $\leq 18$-year-old and $\geq 65$-year-old exhibited the highest median values for both IgM and IgG, with the former being statistically significant. In the undiagnosed group, $13.9 \%$ and $11.1 \%$ were seropositive for IgM and IgG, respectively.

Conclusion IgM and IgG displayed a similar initial increase (within 1/2 weeks), with IgG having a significant decrease after the 21 st week post-diagnosis, translating a loss of immunity at this point. The youngest and oldest symptomatic age groups were found to be the highest responders. Antibody assays enabled the identification of previously undiagnosed participants.
\end{abstract}

Keywords COVID-19 $\cdot$ Immune response $\cdot$ Vaccination $\cdot$ PCR diagnosis $\cdot$ SARS-CoV-2

\section{Introduction}

The last month of 2019 saw the beginning of the biggest global health, economic and social crisis of the twenty-first

Cristina Maria Nunes Almeida

calmeida@ufp.edu.pt

1 FP-ENAS (UFP Energy, Faculty of Health Sciences, Environment and Health Research Unit), Fernando Pessoa University, Oporto, Portugal

2 Dra.Matilde Sampaio Clinical Analysis Laboratory, Mogadouro, Portugal

3 LAQV@REQUIMTE, Oporto University, Oporto, Portugal

4 Centro Hospitalar Tondela, Viseu, Portugal

5 Faculty of Pharmacy, Oporto University, Oporto, Portugal

6 UCIBIO@REQUIMTE, Oporto University, Oporto, Portugal century. In December 2019, Wuhan, China, had just announced the first cases of severe pneumonia caused by the unknown agent later recognized as severe acute respiratory syndrome coronavirus 2 (SARS-CoV-2), a $\beta$-coronavirus responsible for the COVID-19 disease. This RNA virus, the seventh human coronavirus ever to be described, quickly spread to the point of a global pandemic, as declared by the World Health Organization (WHO) on March 11, 2020 [1-3].

Studies published shortly after the onset of the outbreak revealed that a high number of asymptomatic individuals had contributed to the rapid spread between communities and across countries $[4,5]$. Due to the high circulation of people, the virus quickly reached the entire planet, causing considerable morbidity and mortality in the most vulnerable groups, particularly the elderly. By February 2, 2021, there had been 102,817,575 confirmed cases of COVID-19, including 2,227,420 deaths, as reported by WHO [6]. 
Knowledge of the immune response to the infection was and continues to be, of absolute relevance to the understanding of the SARS-CoV-2 infection. The post-infectious humoral response, its efficacy, and longevity have now become essential to help predict future group immunity and, so, guide the rational use of vaccination, as well as the implementation of upcoming pharmacological and social etiquette measures [2, 5].

Early research on the production of specific antibodies resulted in the identification of two structural proteins of SARS-CoV-2 against which antibodies were created: the spike glycoprotein $(\mathrm{S})$ and the nucleocapsid protein $(\mathrm{N})$. The $\mathrm{S}$ protein, a type I transmembrane glycoprotein, mediates entry into human respiratory epithelial cells by binding to the cell surface receptor of the angiotensin-converting enzyme 2 (ACE2) through its receptor-binding domain (RBD) $[2,5]$. The nucleocapsid $(\mathrm{N})$ protein forms complexes with the genomic RNA and interacts with the viral membrane protein, playing a critical role in increasing the efficiency of viral transcription and assembly [2, 7].

It has been suggested that IgG antibodies targeting $\mathrm{S}$ proteins may be more specific, while those targeting the N protein may be more sensitive, particularly in the early stages of infection, despite decreasing post-infection [8].

The persistence of IgG allows for the identification of previously infected, and potentially immune, individuals [9]

The host's immune system reacts to SARS-CoV-2's infection by producing specific $\mathrm{IgG}$ antibodies. These have been reported to appear in the serum from a few days up to 2 weeks after initial symptom onset, when viral RNA is no longer detectable $[10,11]$. According to current literature, there appears to be an emerging consensus that anti-S and anti-N antibody titers vary over time, neutralize rapidly, and may even be absent in infected people [2, 5, 7, 12].

With this knowledge, and considering the shortage of vaccines, what may be the risks of delaying, and even preventing, vaccination in previously infected groups?

This study aimed to assess the immunization status against SARS-CoV-2, via the detection of IgG and IgM antibodies against the virus $\mathrm{N}$ protein, as well as IgM and IgG antibody behavior of a group of 362 unvaccinated participants (including diagnosed and undiagnosed individuals, with and without known virus exposure) that voluntarily sought the lab for testing.

\section{Methods}

\section{Sample selection}

The work was carried out between April 2020 and February 2021 in the Clinical Analysis Laboratory Dra. Matilde Sampaio Lda, Mogadouro, located in Northeast Portugal. The study group consisted of 362 participants who voluntarily sought the laboratory for antibody testing against SARSCoV-2. Of this group, 10 individuals were submitted to a second analysis. The diagnosis of infection was based on a positive reverse transcription polymerase chain reaction test (RT-PCR), using COVID-19 Fast RealAmp Kit (GeneFinder ${ }^{\mathrm{TM}}$ ) of a nasopharyngeal swab sample.

Upon admission, a clinical-epidemiological survey was conducted. Participants were questioned regarding previous history of SARS-CoV-2 infection (including the date of the first positive result, for formerly diagnosed individuals), known exposure to a positive case, and associated symptomatology (namely, fever of $>37.5^{\circ} \mathrm{C}$, cough, myalgias, dyspnoea, rhinorrhea, odynophagia, sneezing, diarrhea, nausea and/or vomiting, anosmia, and dysgeusia and/or ageusia). All data concerning the clinical-epidemiological surveys was manually uploaded to our servers by each patient, subsequently encrypted, and then linked to the corresponding analytical report. Only the Clinical Director or a Delegate was allowed access to these reports (under the obligation of professional secrecy, as well as in compliance with all integrity and protection laws in force). Informed written consent was obtained from all participants before the collection of any data regarding the study.

Analytical tests were carried out using Abbott's ARCHITECT iSystem i1000 equipment with a chemiluminescent microparticle immunoassay (CMIA) in serum samples. The ARCHITECT System calculates the medium chemiluminescence calibrator value from 3 replicates of the calibrator (C) and stores the result. The sample results are calculated by dividing the sample (S) by the calibrator. The default result units for this SARS-COV-2 IgG essay are the index (S/C).

We quantitatively measured $\operatorname{IgG}$ and $\operatorname{IgM}$ antibodies using a commercial Abbott kit. According to the manufacturer's instructions, IgG results were deemed positive if the cut-off index $(\mathrm{S} / \mathrm{C})$ was $\geq 1.4$, while negative results were defined by a cut-off of $<1.4$; as for IgM, results were considered positive if $>1.1$, borderline if $\geq 0.8$ to 1.1 , and negative if $<0.8$.

At the time of this study, the available tests in Portugal only detected IgG and IgM antibody classes against the $\mathrm{N}$ antigen.

\section{Statistical methods}

Graphical presentations and statistical analysis were performed using SPSS ${ }^{\circledR}$ Statistics version 26.0 (IBM, Armonk, NY, USA), considering a significance level of 0.05 for all statistical inference situations. Counts and proportions ( $n$ [\%]) were reported for categorical variables, and quantitative data was described as median values and corresponding 25th and 75th percentiles (med [interquartile range]), as all variables were non-normally distributed. Proportions 
were assessed using the chi-square test or Fisher's exact test, whenever appropriate. Comparison of quantitative data among categories of the most relevant covariates used the Mann-Whitney $U$ or Kruskal-Wallis $H$, whenever appropriate. For related samples, the Wilcoxon test was used.

\section{Results}

Characterization of the total sample $(n=362)$ is shown in Table 1 . The population was composed of mostly women (63.0\%), and the median age was 42 years (between 31.0 to 53.5). Of those who answered, 114 reported a previous diagnosis of SARS-CoV-2 infection by RT-PCR, 133 had a positive history of contact with an infected individual, while the remainder did not or did not know. Regarding clinical presentation, $62(19.3 \%)$ reported symptoms associated with COVID-19 (mainly fever, cough, muscle pain, lack of smell, and taste). The infections/exposures happened between February 2020 and January 2021, and the study was conducted between April 2020 and February 2021. In the infected group, analyses were performed 33.5 to 112.5 days (IQR values) after diagnosis (median of 60 days). The clinical and biologic comparisons between the SARS-CoV-2 diagnosed group $(n=115,31.8 \%)$ and the non-infected/undiagnosed group $(n=247,68.2 \%)$ are presented in Table 2. Comparing both groups, there were no statistical differences found between genders and across age categories. The SARSCoV-2 diagnosed group reported a significantly higher number of symptoms, positive exposures, and exhibited higher values of both IgM and IgG antibodies (median values and \% of seropositive). Nonetheless, of these, 37 (44.6\%) and $32(27.8 \%)$ did not present with positive $\operatorname{IgM}$ and $\operatorname{IgG}$ antibodies, respectively. Whereas in the undiagnosed group, $16(13.9 \%)$ were seropositive for $\operatorname{IgM}$ and 27 (11.1\%) for $\mathrm{IgG}$ (Table 2), with 13 being both $\operatorname{IgM}$ and $\mathrm{IgG}$ positive.

The immunologic characterization of the SARS-CoV-2 infected group $(n=114)$ is shown in both Table 3 and Fig. 1a-c.

For IgM, peak concentrations occurred within the first 3 weeks of infection. A median value of 3.7 was achieved during the 2 nd week (with $83.3 \%$ of seropositive), while a peak of 5.8 took place in the 3rd week (with $85.7 \%$ of seropositive), followed by a consistent decrease in values, accentuated after the 6th week (with $42.1 \%$ of seropositivity between the 7 th and 10th weeks). IgM values were also found to be significantly different across age groups $(p=0.046)$, with the 18 -year-old or under and the 65-yearold or higher having the highest IgM values. The number of weeks between diagnosis and serologic testing did not differ significantly across ages groups $(p=0.489)$. Nonetheless, the 65-year-old or older group was the first to be tested (about 5.5 median weeks after diagnosis), followed by the age groups of 19-40 and 41-64 (9 median weeks after diagnostic) and, finally, by the 18 years or younger (with 15.0 median weeks after diagnostic). The symptomatic group had higher IgM values than the asymptomatic group (3.0 vs. 0.7, respectively, $p=0.018$ ). Fever, the most reported symptom,
Table 1 Clinical and biological characterization of the sample $(n=362)$

\begin{tabular}{ll}
\hline Variable & Total study sample \\
\hline Gender $(n=362), n(\%)$ & $228(63.0)$ \\
Female & $134(37.0)$ \\
Male & $42(31.0-53.5)$ \\
Age (years) $(n=361)$, med $(\mathrm{IQR})$ & \\
Age categories (years) $(n=361), n(\%)$ & $19(5.3)$ \\
$\leq 18$ & $152(42.1)$ \\
$19-40$ & $98(43.5)$ \\
$41-64$ & $18(9.1)$ \\
$\geq 65$ & \\
SARS-CoV-2 infection $(n=361), n(\%)$ & $114(31.7)$ \\
Yes & $247(68.3)$ \\
No & February 2020 to January 2021 \\
SARS-CoV-2 infection period $($ date $)(n=106)$, min-max & April 2020 to February 2021 \\
Analysis period (date) $(n=360), \min -$ max & $60.0(33.5-110.0)(7-340)$ \\
Days from diagnosis till analysis $(n=105)$, med (IQR) $(\min -$ max $)$ & \\
Exposure to a positive case $(n=337), n(\%)$ & $133(39.5)$ \\
Yes & $204(60.5)$ \\
No/Do not know & \\
Symptoms of COVID-19 $(n=318), n(\%)$ & $62(19.3)$ \\
Yes (one or more) & $256(79.8)$ \\
No &
\end{tabular}

Med (IQR): median (interquartile range); $n(\%)$ : count (percentage); min-max: minimum-maximum 
Table 2 Clinical and biological characterization of the sample with and without SARS-CoV-2 infection $(n=362)$

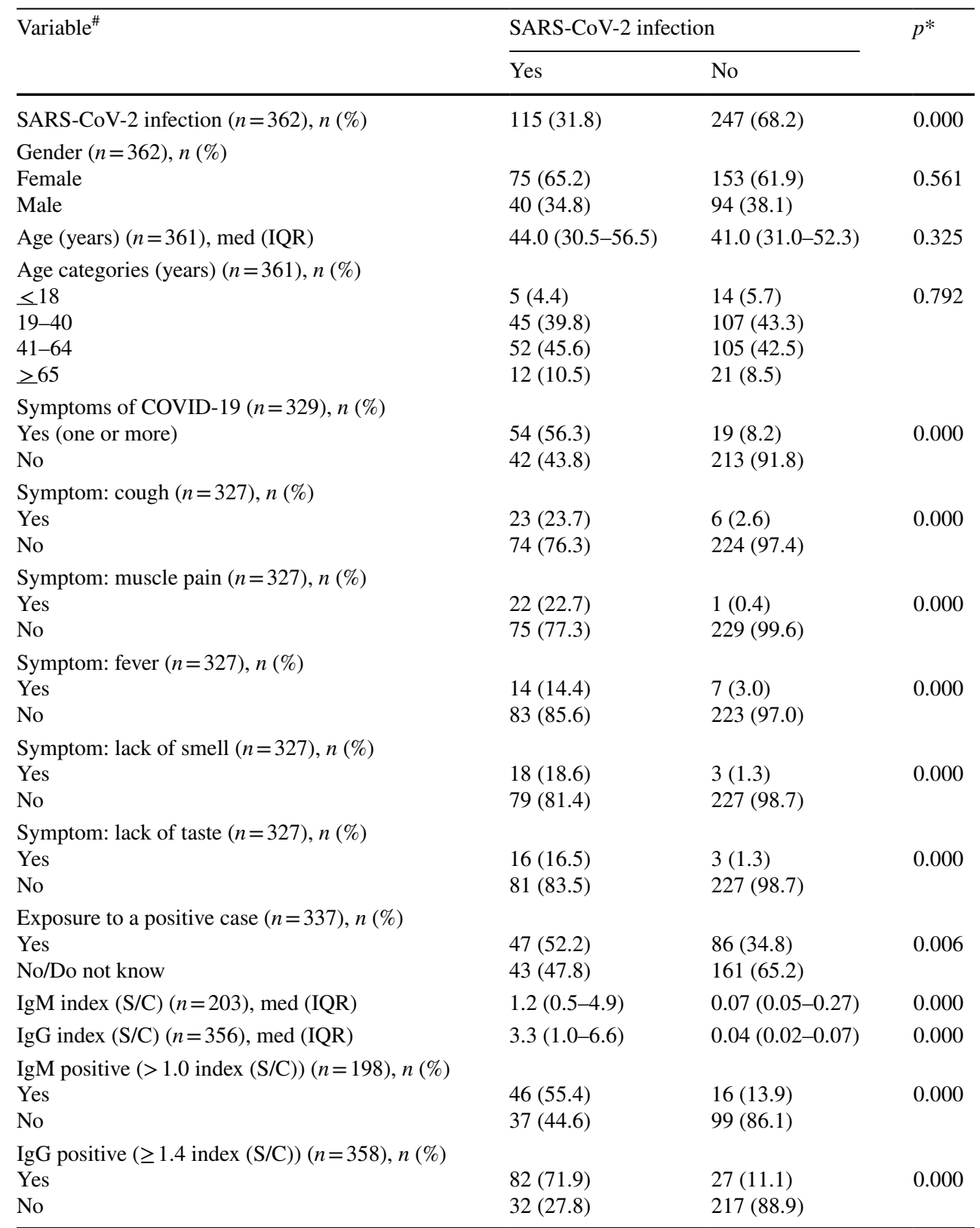

Med (IQR): median (interquartile range); $n(\%)$ : count (percentage)

*Mann-Whitney or chi-square test or Fisher's exact test

${ }^{\#}$ For each variable, the values of $n$ correspond to the total number of answers/results was found to be positively associated with $\operatorname{IgM}(4.4$ vs 1.1 average $\operatorname{IgM}$ values in people with and without fever, respectively, $p=0.029)$. Furthermore, IgM values were significantly higher in individuals infected in 2021 versus 2020 (4.0 vs $1.1, p=0.026$ ); however, the former were analyzed significantly sooner, when comparing with the 2020 group (32 vs 60 median days after infection, respectively).

IgG values did not reveal statistically significant differences between gender, age categories, year of infection, or symptomatic/asymptomatic groups. In the first 2 weeks following COVID-19 diagnosis, IgG showed a significant increase (median value of 2.9 and $60.0 \%$ of seropositive), reaching a peak of 7.9 (corresponding to a peak) during the 3 rd week (with $85.7 \%$ of seroconversion at this point). After that, antibody count decreased, particularly between the 4th-6th and $7 \mathrm{~h}-10$ th weeks. Despite this decrease, $75 \%$ were still $\operatorname{IgG}$ seropositive after the 10th week (Table 3 ). Analyzing in detail the values after 10 weeks, it was possible to detect that the median IgG decreased to a seronegative 
Table 3 Immunologic characterization of the SARS-CoV-2 infected group $(n=114)$

\begin{tabular}{|c|c|c|c|c|c|c|}
\hline \multirow[t]{2}{*}{ Variable } & \multicolumn{3}{|l|}{$\operatorname{IgM}$} & \multicolumn{3}{|l|}{$\operatorname{IgG}$} \\
\hline & $\%(\mathrm{n})$ seropositive & Med (IQR) (n) & $\mathrm{p}^{*}$ & $\%(n)$ seropositive & Med (IQR) (n) & $\mathrm{p}^{*}$ \\
\hline \multicolumn{7}{|l|}{ Weeks after diagnosis (categories) } \\
\hline $1-2$ & $83.3(n=5)$ & $3.7(0.85-7.0)(n=7)$ & 0.002 & $60.0(n=6)$ & $2.9(0.07-4.4)(n=10)$ & 0.017 \\
\hline 3 & $85.7(n=6)$ & $5.8(3.1-15.1)(\mathrm{n}=7)$ & & $85.7(n=6)$ & $7.9(3.3-9.3)(\mathrm{n}=7)$ & \\
\hline $4-6$ & $72.7(n=16)$ & $2.1(0.8-12.4)(n=23)$ & & $80.8(n=21)$ & $4.3(1.5-7.9)(n=26)$ & \\
\hline $7-10$ & $42.1(\mathrm{n}=8)$ & $0.7(0.2-2.2)(n=20)$ & & $57.1(\mathrm{n}=12)$ & $2.1(0.2-5.4)(n=21)$ & \\
\hline$>10$ & $31.8(\mathrm{n}=7)$ & $0.7(0.2-1.5)(\mathrm{n}=23)$ & & $75.0(\mathrm{n}=30)$ & $3.1(1.2-5.2)(n=40)$ & \\
\hline \multicolumn{7}{|l|}{ Age categories (years) } \\
\hline$\leq 18$ & $50.0(\mathrm{n}=1)$ & $3.5(0.7-3.5)(n=2)$ & 0.046 & $100.0(n=5)$ & $4.6(3.9-7.2)(\mathrm{n}=5)$ & 0.113 \\
\hline 19-40 & $51.4(\mathrm{n}=19)$ & $0.97(0.38-3.9)(n=39)$ & & $64.4(n=29)$ & $2.2(0.62-4.2)(n=45)$ & \\
\hline $41-64$ & $51.5(\mathrm{n}=17)$ & $0.92(0.35-4.8)(n=34)$ & & $74.5(n=38)$ & $3.6(1.0-7.0)(\mathrm{n}=50)$ & \\
\hline$>65$ & $90.0(n=9)$ & $7.5(1.4-18.6)(n=10)$ & & $75.0(\mathrm{n}=9)$ & $5.5(1.1-8.9)(\mathrm{n}=12)$ & \\
\hline \multicolumn{7}{|l|}{ Symptoms of COVID-19 } \\
\hline Yes (one or more) & $64.7(n=22)$ & $3.0(0.70-5.7)(n=36)$ & 0.018 & $75.0(n=33)$ & $3.8(1.5-7.9)(n=42)$ & 0.135 \\
\hline No & $43.5(n=10)$ & $0.7(0.23-1.9)(n=24)$ & & $71.4(\mathrm{n}=30)$ & $3.2(1.0-4.8)(n=44)$ & \\
\hline \multicolumn{7}{|l|}{ Year of the infection } \\
\hline 2020 (60 median days after infection) & $53.0(n=35)$ & $1.1(0.3-4.4)(n=69)$ & 0.026 & $69.9(n=65)$ & $3.2(1.0-6.6)(n=93)$ & 0.094 \\
\hline 2021 (32 median days after infection) & $72.7(\mathrm{n}=8)$ & $4.0(0.8-11.7)(n=12)$ & & $91.7(n=11)$ & $4.1(1.7-9.2)(n=12)$ & \\
\hline \multicolumn{7}{|l|}{ Gender } \\
\hline Female & $51.0(n=26)$ & $0.9(0.4-4.0)(n=54)$ & 0.186 & $70.7(n=53)$ & $3.8(0.7-6.5)(n=75)$ & 0.913 \\
\hline Male & $62.5(n=20)$ & $2.0(0.6-5.4)(n=32)$ & & $74.4(n=29)$ & $2.5(1.3-7.7)(n=38)$ & \\
\hline
\end{tabular}

Med (IQR) (n): median (interquartile range) (count); \% (n): percentage (count)

*Mann-Whitney or Kruskal-Wallis test

value after 21 weeks (Fig. 1c), with only $33.3 \%$ IgG seropositive remaining after this time.

$\operatorname{IgM}$ and $\operatorname{IgG}$ antibody behavior for asymptomatic and symptomatic groups is represented in Fig. 2a, b. As depicted, symptomatic patients had a higher immune response when compared to their asymptomatic peers, especially during the 3rd and 6th weeks after diagnosis.

In the group without a previous COVID-19 diagnosis ( $n=247$ persons, $68.3 \%$ of our total sample), $16(14.0 \%)$

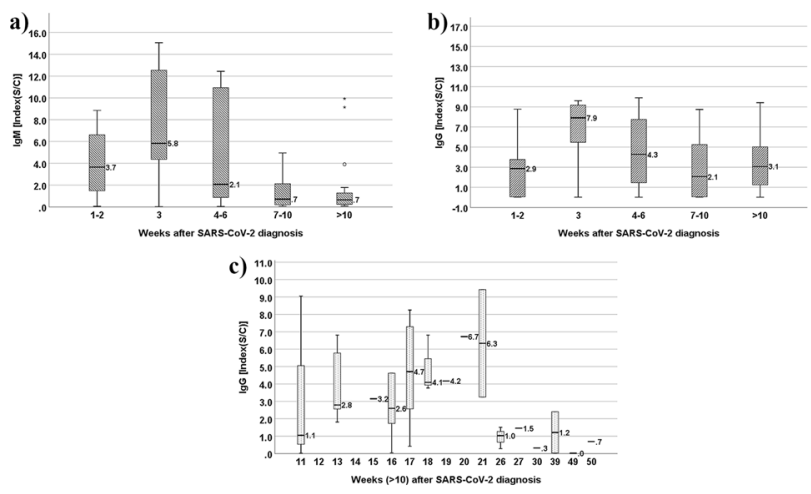

Fig. 1 a Evolution of patient $\operatorname{IgM}$ values (median (IQR)) in the 10 weeks following SARS-CoV-2 diagnosis. b Evolution of patient IgG values (median (IQR)) in the 10 weeks following SARS-CoV-2 diagnosis. c Evolution of patient IgG values (median (IQR)) past the 10th week following SARS-CoV-2 diagnosis were IgM positive, 27 (11.1\%) were IgG positive, and 13 were both $\operatorname{IgM}$ and $\operatorname{IgG}$ positive.

To gain further understanding of antibody response in COVID-19 infection, we analyzed 2 sequential samples, an initial (t1) and a follow-up (t2) one, in a small group of case-patients (total $n=10$ ), including 2 individuals aged 18 years or under, 5 between the ages of 19 to 40 years, and 3 between the age of 41 to 64 . T1 took place, respectively, 3.5 and 5 weeks after diagnosis for $\operatorname{IgM}$ and $\mathrm{IgG}$, while t2 happened approximately 5 to 6 weeks after (Table 4 ). Between $\mathrm{t} 1$ and $\mathrm{t} 2$, IgM median values decreased significantly to about half of their initial value (4.3 vs 2.3 median values, respectively) ( $p=0.012$ ), while $\operatorname{IgG}$ median values increased (3.8 to 4.6), although not significantly. There was
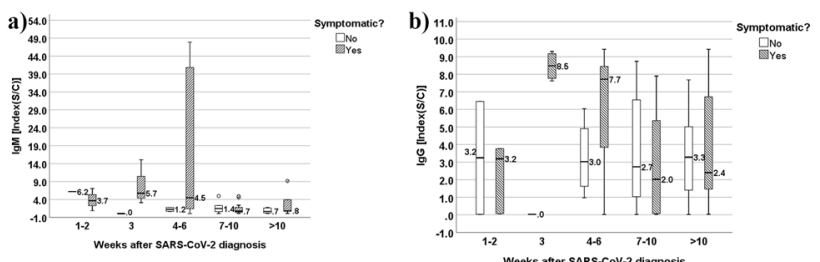

Fig. 2 a Evolution of IgM values (median (IQR)) in the 10 weeks following SARS-CoV-2 diagnosis in symptomatic (cough, muscle pain, fever, lack of smell, and taste) and non-symptomatic patients. b Evolution of IgG values (median (IQR)) in the 10 weeks following SARS-CoV-2 diagnosis in symptomatic (cough, muscle pain, fever, lack of smell and taste) and non-symptomatic patients 
Table 4 IgM and IgG titer evolution from time 1 to time 2 after diagnostic in a SARS-CoV-2 infected group (total $n=10$ )

\begin{tabular}{|c|c|c|c|c|c|c|c|}
\hline \multirow{2}{*}{$\begin{array}{l}\text { IgM collection } \\
\text { time (median (IQR) } \\
\text { weeks) }\end{array}$} & \multicolumn{3}{|l|}{$\operatorname{IgM}$} & \multirow{2}{*}{$\begin{array}{l}\text { IgG collection time } \\
\text { (median (IQR) } \\
\text { weeks) }\end{array}$} & \multicolumn{3}{|l|}{$\mathrm{IgG}$} \\
\hline & $\%(n)$ seropositive & Med (IQR) $(n)$ & $p^{*}$ & & $\%(n)$ seropositive & Med (IQR) (n) & $p^{*}$ \\
\hline $3.5(2.0-8.3)(\mathrm{t} 1)$ & $87.5(n=7)$ & $4.3(2.5-9.1)(n=8)$ & 0.012 & $5.0(2.0-10.8)(\mathrm{t} 1)$ & $90.0(n=9)$ & $3.8(2.4-6.5)(n=10)$ & 0.444 \\
\hline $9.4(4.4-14.1)(\mathrm{t} 2)$ & $87.5(n=7)$ & $2.3(1.5-7.7)(n=8)$ & & $10.0(4.8-17.0)(\mathrm{t} 2)$ & $80.0(n=8)$ & $4.7(1.3-6.1)(n=10)$ & \\
\hline
\end{tabular}

Med (IQR) (n): median (interquartile range) (count); \% (n): percentage (count)

*Related-samples Wilcoxon test

considerable percentage of $\operatorname{IgM}$ and $\operatorname{IgG}$ seropositivity in both collection times (Table 4).

\section{Discussion}

SARS-CoV-2 is the novel coronavirus responsible for the COVID-19 worldwide outbreak, and for which timing, magnitude, and longevity of humoral immunity is not yet well understood. Naturally, it is therefore important to define parameters in which antibody tests can provide meaningful data in the absence of PCR testing.

In our study, we tested a population of 362 unvaccinated individuals for both $\operatorname{IgM}$ and $\operatorname{IgG}$ antibodies against the SARS-CoV-2 $\mathrm{N}$ antigen. Of those who answered, 114 $(31.7 \%)$ had a previous history of diagnosis.

In accordance with others $[1,7,13]$, most of our case patients were middle aged adults $(85.6 \%)$, averaging at 42 years old (age category between 19 to 64 years old). Only $5.3 \%$ were 18 years or younger, while $9.1 \%$ were aged 65 or older. The median of days between diagnosis and analysis was 60 (about 8.5 weeks). In the infected group, only 51.7\% presented with characteristic COVID-19 symptoms (namely, cough, muscle pain, fever, lack of smell and taste) (Table 2).

These findings are in accordance with others [1, 4], who report that most cases are classified as either asymptomatic or mild [1]. Similar to our study, some reported that half of the individuals with positive test results did not present with any symptoms at the time of testing $[4,14,15]$.

Several publications $[2,7,8,16-18]$ described that most individuals infected with the new coronavirus displayed an antibody response within 1 to 2 weeks after infection, followed by an expected decrease in antibody count, the degree of which varied in accordance with the severity of the case in question [5, 13]. Seow J. et al. [7] described that antibodies begin waning following 40 days (about 6 weeks) after the onset of symptoms and may even be negative for cases with a severity score of 0 ; other authors [2] reported reinfection with a homologous coronavirus after as little as 80 days. Cao et al. [19] reported waning after about 12 to 34 months postinfection in some individuals with SARS-CoV-2 infection; however, the exact antibody response is, at this date, still unknown. It has been also observed that anti-N antibodies, although more sensitive, waned earlier than anti-S antibodies [8], a finding which may have impacted our study, where only anti-N antibodies were researched.

Accordingly, in our study, the participants with proven SARS-CoV-2 infection displayed IgM and IgG antibody response (with seroconversion) within the initial 1 to 2 weeks after infection (Table 3 ). After this time, antibody count continued to increase reaching a peak in the 3 rd week, as expected after acute viral infection [7]. In the following weeks, values decreased, particularly after the 6th week, achieving a median seronegative value for IgM after this collection time point (Table 3). Despite the reduction, median IgG values were still positive 21 weeks (approximately 5 months) after diagnosis, decreasing to a seronegative median value after this time point only (Fig. 1b, c). Similar findings were reported by Mai, H. K., et al. and Wajnberg, A., et al., where IgG values remained positive until 4.2 and 5 months, respectively. In the study presented by McDade et al., seronegativity rates in the early convalescent phase reached $40 \%$ in asymptomatic patients, but only $12.9 \%$ in symptomatic ones [14]. All authors agreed these findings may prove useful in future vaccine development, as well as immunity strategy and serological surveys.

Regarding the diagnosed group, the majority ( $n=26$, $70.3 \%$ ) of the participants without seroconversion for IgM $(n=37)$ (Table 2$)$ only sought the lab for serologic testing after the 6th week post-diagnosis, and, thus, were already in the waning phase. Of the rest, 3 do not remember the date of diagnosis of SARS-CoA-2 infection and 8 visited the lab within the first 3 weeks after diagnosis.

Of the 32 with diagnosed SARS-CoV-2 infection (27.8\%) but without IgG seroconversion (Table 2), 6 (about 20\%) only pursued the lab past the 21 st week post-diagnosis, thus suggesting they had already lost their immunity. Similar to the other group, 3 participants missed the diagnosis date (and so we are unaware of their status at the time of the analysis). The remainder visited the lab during the 2 nd and 17 th week following diagnosis, and so, in accordance with our findings, would have been expected to have had positive values for $\operatorname{IgG}$ at this time. 
It is described that in some mild cases [2, 18], detection of antibodies may require a long time after symptom onset, and, in a small number of cases, may not occur at all. A recent study [20] also showed that the antibody titers of patients with mild coronavirus disease declined more rapidly when compared with those presenting with severe acute respiratory syndrome, but it is remains unclear why neutralizing antibody response appears to correlate with disease severity [21]. On one hand, a higher viral load may lead to more severe disease and generate a stronger antibody response through increased levels of viral antigen. On the other hand, antibodies could have a causative role in disease severity, although there is currently no evidence for antibody-dependent enhancement in COVID-19 [22]. Also, these milder cases are reported as having lower immunity, and so, a presumably higher susceptibility to reinfection [2]. Accordingly, our asymptomatic case-patients $(n=42$, 48.3\%) (Table 2) displayed lower median values for both $\mathrm{IgM}$ and $\mathrm{IgG}$ antibodies, although only significant in the case of the former (Table 3). Asymptomatic cases can justify the lower IgM and IgG values. In fact, looking into the behavior of IgM and IgG in symptomatic $v s$ asymptomatic groups over the weeks after diagnosis (Fig. 2a, b), it suggested that symptomatic individuals appeared to have a more intense immune response, particularly from the 3rd to the 6th weeks following diagnosis. Nonetheless, out of the 8 individuals without IgM seroconversion who sought the lab within the first 3 weeks after diagnosis, 7 were symptomatic. Similarly, regarding seroconversion for $\mathrm{IgG}$, out of 23 who visited the lab before the 21 st week after diagnosis, our proposed immunization cut-off timing, 14 (60.9\%) were also symptomatic. Hence, the existence of a causal relationship between symptomatology and seroconversion remains nonconsensual, as reported by Choe PG et al. [5] who stated that neutralizing antibody titers decreased more rapidly in symptomatic versus asymptomatic patients. However, this unusual behavior was justified in this study by the age of the symptomatic participants, who were considerably older than their asymptomatic counterparts.

In our work, the $\leq 18$-year-old and $\geq 65$-year-old age groups were associated with the higher median values for both $\operatorname{IgM}$ and $\operatorname{IgG}$, with this result being significant for IgM only $(p=0.046)$ (Table 3$)$. Although the number of weeks after SARS-CoA-2 infection is enough for the seroconversion of both IgM and IgG (according to our study) in both age groups, because the analysis were performed after a median of 15 weeks (for $\leq 18$-year-old) and of 5.5 weeks (for $\geq 65$-year-old) after the diagnosis, it would be maybe too late to still detect changes in IgM, mainly in the younger group (because our data showed an accentuated decrease of IgM values after the 6th week). Nevertheless, that was not the case, which may be in accordance with a more reactive immune system associated with a younger age [23].
Regarding the older group, the detected values may be explained by the earlier timing of analysis, which coincided with our found peak in both antibody titers. Further confirmation of this behavior is needed, given the small number of participants present in these age groups.

In this study, the group of participants infected in 2021 displayed higher values of $\operatorname{IgM}$ and $\operatorname{IgG}$, when compared with those infected in 2020, a finding which was significant for IgM only ( $p=0.026$ ) (Table 3 ). This result could be a result of with different, possibly more aggressive, COVID19 strains, first identified in the USA in December 2020 [24], or simply associated with the timing of testing, which took place significantly sooner in 2021 versus 2020 (about 5 vs 9 weeks for 2021 and 2020, respectively, $p=0.000$ ).

Regarding the small group who was submitted to a follow-up analysis, composed of 2 individuals with ages 18 or lower, 5 between 19 and 40, and 3 between 41 and 64 years, antibody behavior was in accordance with previous results for the same time periods (Table 3; Fig. 1), with the exception of $\operatorname{IgM}$ in $\mathrm{t} 2$, which was significantly higher than expected (9.4 median weeks after diagnosis) (Table 4). Analyzing the data, we observed that the 2 individuals who contributed the most to this behavior were two symptomatic women, aged 24 and 61 years old. These findings further reinforce the conclusion that the younger and older age groups may be the highest immune responders; however, further investigation is needed to corroborate this hypothesis.

\section{Conclusions}

The neutralizing antibody titer required for protection from reinfection is not yet known. In our study, we found a seroconversion rate of, respectively, $83.3 \%$ and $60.0 \%$ for $\operatorname{IgM}$ and $\mathrm{IgG}$ within the initial 1st/2nd weeks after diagnosis, with both antibodies reaching peak values within the 3rd week. Antibody count decreased significantly in the following weeks passing the point of seronegativity after the 6th week for IgM the 21 st week for IgG.

All age categories within the diagnosed group displayed antibody production; nevertheless, the groups of 18 years old or younger and 65 years old or older had the highest antibody titers for both IgM and IgG, with the former being statistically significant. However, in the case of this last group, findings could be associated with an earlier timing of analysis.

Symptomatic individuals exhibited higher IgM and IgG values, when compared to asymptomatic ones. Fever was the most reported symptom and showed a positive association with $\operatorname{IgM}$ values.

Regarding the undiagnosed group, at the time of the sample collection, $25 \%$ displayed antibody production, thus 
revealing a previously unknown history of SARS-CoV-2 infection, which we can infer might have added to uncontrolled propagation of the disease.

Finally, the absence of seroconversion in some cases of infection, the low titers of anti-N protein antibodies in general (even lower in asymptomatic cases), and the short durability of specific IgG antibodies (about 21 weeks) strongly suggest that this type of natural immunization might not be able to replace vaccination, particularly not after a period greater than 5 months. However, further studies are needed to help clarify this hypothesis.

Author contribution MD, ID, and CA were major contributors to writing the manuscript. CA performed also the statistical analysis. MD, FM, MP, SJ, and MP performed the analytical tests and conducted the application of the clinical-epidemiological survey. MD, ID, IR, and CA checked and revised the manuscript. All authors read and approved the final manuscript.

Data availability The datasets generated during and/or analyzed during the current study are available from the corresponding author on reasonable request.

Code availability Not applicable.

\section{Declarations}

Ethics approval Not applicable.

Consent to participate Informed written consent was obtained from all participants before the collection of any data regarding the study.

Consent for publication All authors accept the terms and conditions of the editorial for publication.

Conflict of interest The authors declare no competing interests.

\section{References}

1. Wu Z, McGoogan JM (2020) Characteristics of and important lessons from the coronavirus disease 2019 (COVID-19) outbreak in China: summary of a report of 72314 cases from the Chinese Center for Disease Control and Prevention. JAMA 323(13):12391242. https://doi.org/10.1001/jama.2020.2648

2. Kellam P, Barclay W (2020) The dynamics of humoral immune responses following SARS-CoV-2 infection and the potential for reinfection. J Gen Virol 101(8):791-797. https://doi.org/10.1099/ jgv.0.001439

3. Organization (2020) W.H. WHO Director-General's opening remarks at the media briefing on COVID-19 - 11 March 2020. 2020 February 25]; Available from: https://www.who.int/directorgeneral/speeches/detail/who-director-general-s-opening-remarksat-the-media-briefing-on-covid-19---11-march-2020

4. Kimball A et al (2020) Asymptomatic and presymptomatic SARS-CoV-2 infections in residents of a long-term care skilled nursing facility - King County, Washington, March 2020. MMWR Morb Mortal Wkly Rep 69(13):377-381. https://doi. org/10.15585/mmwr.mm6913e1
5. Choe PG et al (2021) Waning antibody responses in asymptomatic and symptomatic SARS-CoV-2 infection. Emerg Infect Dis 27(1):327-329. https://doi.org/10.3201/eid2701.203515

6. WHO (2021) W.H.o. Weekly epidemiological update - 2 February 2021. 2021; Available from: https://www.who.int/publications/ $\mathrm{m} /$ item/weekly-epidemiological-update---2-february-2021

7. Seow J et al (2020) Longitudinal observation and decline of neutralizing antibody responses in the three months following SARS-CoV-2 infection in humans. Nat Microbiol 5(12):15981607. https://doi.org/10.1038/s41564-020-00813-8

8. Fenwick C et al (2021) Changes in SARS-CoV-2 spike versus nucleoprotein antibody responses impact the estimates of infections in population-based seroprevalence studies. J Virol 95(3):e01828-e1920. https://doi.org/10.1128/jvi.01828-20

9. Shen $C$ et al (2020) Treatment of 5 critically ill patients with COVID-19 with convalescent plasma. JAMA 323(16):15821589. https://doi.org/10.1001/jama.2020.4783

10. Zhao J et al (2020) Antibody responses to SARS-CoV-2 in patients with novel coronavirus disease 2019. Clin Infect Dis 71(16):2027-2034. https://doi.org/10.1093/cid/ciaa344

11. Xiao AT, Gao C, Zhang S (2020) Profile of specific antibodies to SARS-CoV-2: The first report. J Infect 81(1):147-178. https://doi.org/10.1016/j.jinf.2020.03.012

12. Choe PG et al (2017) MERS-CoV antibody responses 1 year after symptom onset, South Korea, 2015. Emerg Infect Dis 23(7):1079-1084. https://doi.org/10.3201/eid2307.170310

13. Sun B et al (2020) Kinetics of SARS-CoV-2 specific IgM and IgG responses in COVID-19 patients. Emerg Microbes Infect 9(1):940-948

14. McDade TW et al (2021) Symptoms of COVID-19 infection and magnitude of antibody response in a large community-based study. medRxiv:2021.02.04.21251170. https://doi.org/10.1101/ 2021.02.04.21251170

15. Mai HK et al (2021) Long-term humoral immune response in persons with asymptomatic or mild SARS-CoV-2 infection. Vietnam Emerging infectious diseases 27(2):663-666. https:// doi.org/10.3201/eid2702.204226

16. Hsueh PR et al (2004) Chronological evolution of IgM, IgA, $\mathrm{IgG}$ and neutralisation antibodies after infection with SARSassociated coronavirus. Clin Microbiol Infect 10(12):1062-1066

17. Yan HP et al (2006) A follow up study of total IgM, IgG, nucleoprotein and spike protein antibodies against severe acute respiratory syndrome (SARS) coronavirus in patients with SARS. Zhonghua Nei Ke Za Zhi 45(11):896-899

18. Wan Y et al (2020) Performance verification of anti-SARSCoV-2-specific antibody detection by using four chemiluminescence immunoassay systems. Ann Clin Biochem 57(6):429-434

19. Cao WC, Liu W, Zhang PH, Zhang F, Richardus JH (2007) Disappearance of antibodies to SARS-associated coronavirus after recovery. N Engl J Med 357:1162-1163

20. Ibarrondo FJ, Goodman-Meza FJ, Elliott D, Hofmann J, Hausner MA C et al (2020) Rapid decay of anti-SARS-CoV-2 antibodies in persons with mild Covid-19. N Engl J Med 383:1085-1087. https://doi.org/10.1056/NEJMc2025179

21. Lee, N.e.a. (2006) Anti-SARS-CoV IgG response in relation to disease severity of severe acute respiratory syndrome. J Clin Virol 35:179-184

22. Iwasaki AY (2020) The potential danger of suboptimal antibody responses in COVID-19. Nat Rev Immunol 20:339-341

23. Lawton $\mathrm{G}$ (2020) You're only as young as your immune system. New Sci 245(3275):44-48

24. https://www.cdc.gov/coronavirus/2019-ncov/cases-updates/ variant-surveillance/variant-info.html

Publisher's Note Springer Nature remains neutral with regard to jurisdictional claims in published maps and institutional affiliations. 